\title{
Thrombolysis for PE: less is more?
}

\section{Luke S Howard}

Despite the significant advances in diagnostic imaging, risk stratification and anticoagulants over the past two decades in the field of PE, very little progress has been made in clarifying the role of thrombolysis in the treatment of submassive or intermediate-risk PE. Perhaps the simplest illustration of our uncertainty is that guideline committees struggle to come up with consistent recommendations. While the National Institute for Health and Care Excellence (NICE) Clinical Guideline 144 makes it clear that thrombolysis should not be administered to patients outside the context of haemodynamic instability, ${ }^{1}$ the European Society of Cardiology (ESC) guidelines leave open the possibility that we 'consider' reperfusion therapy in some patients with intermediate-high risk PE. ${ }^{2}$ While NICE guidance does not perhaps reflect the complexity of different presentations of PE, the ESC guidelines leave us with a great deal of uncertainty when making major clinical decisions.

Large randomised controlled trials have not been able to provide a clear answer; rather they leave us with further hypothesis-generating conclusions. The Pulmonary Embolism Thrombolysis (PEITHO) trial randomised over 1000 patients with ESC-defined intermediate-high risk PE (haemodynamically stable, right ventricular dysfunction on imaging and positive troponin) to placebo or tenecteplase. ${ }^{3}$ One would imagine that with a positive primary endpoint, this would have provided clear direction; however, it did not. This is because the primary endpoint was a composite of mortality and haemodynamic collapse at 7 days and, while relevant, this does not take account of the significant morbidity burden associated with bleeding in the tenecteplase arm. Restricting the outcome to mortality alone at 30 days failed to show a benefit of tenecteplase over placebo, since a reduction in PE-related mortality was counterbalanced by an increase in bleeding-related deaths.

With this equipoise in mortality, why, then, is there still a search for a role for thrombolysis in selected

National Heart \& Lung Institute, Imperial College London, London, UK

Correspondence to Dr Luke S Howard, National Pulmonary Hypertension Service, Hammersmith Hospital, Imperial College Healthcare NHS Trust, London W12 OHS, UK; I.howard@imperial.ac.uk haemodynamically stable patients? First, we recognise that there is still a significant mortality risk associated with this group of patients and more needs to be done. Overall mortality at 30 days in PEITHO was lower than expected, at under $3 \%$. $^{3}$ A recent study of approximately 900 patients showed that the 30-day mortality for patients with intermediate-high risk PE was $7.7 \%$, of which $4.8 \%$ was PE-related. ${ }^{4}$ This is not an isolated finding and it is not clear why there was this difference. It may relate to a reluctance to recruit 'sicker' patients with intermediate-high risk PE to trials if they can be offered thrombolysis outside a trial. Alternatively, patients may be monitored more closely for deterioration in trials.

Second, we may be able to improve the benefit:risk profile of reperfusion therapy with better patient selection. While PEITHO used the intermediate-high risk patient group, recent data have suggested that this group of patients does not have a significantly higher mortality than the intermediate-low risk patients. ${ }^{4}$ Various studies have shown that combinations of risk factors that include biomarkers and deep vein thrombosis identified on leg vein Doppler may identify normotensive patients at the highest risk of adverse outcomes who would benefit from reperfusion. ${ }^{56}$ Furthermore, a deeper look into the PEITHO data shows that younger patients were at lower risk of adverse events. Of the 13 patients who suffered a stroke in the study, only one was under the age of $65 .^{3}$ This was further supported by the results of a meta-analysis demonstrating that the risk of major bleeding was significantly attenuated in patients aged 65 or under receiving thrombolysis. ${ }^{7}$

Third, we may be able to make thrombolysis safer by reducing the dose and/ or delivering it centrally via catheter. Several trials have examined the question of adjusting the dose or method of delivery of thrombolytic therapy, both comparing it against placebo or full-dose thrombolysis. Different entry criteria and a lack of a clear understanding of whether full-dose thrombolysis is better than placebo hamper conclusions from these small to medium-sized studies. In Thorax, Jimenez and colleagues present a systematic review and network meta-analysis of all forms of thrombolysis in an attempt to provide some clarity from this fog of heterogeneous studies. ${ }^{8}$ They conclude that thrombolysis does not seem to offer a clear advantage compared with standard anticoagulation; however, a low dose may be the best option to consider when thrombolysis is being used.

It is worth exploring these interesting findings in more detail. The authors were not able to separate out high-risk (haemodynamic instability) patients from non-high-risk patients. Thrombolysis is still recommended in high-risk patients. Naturally, including these patients in the overall analysis may have contaminated the overall findings; however, since they were negative, this criticism is invalid. It should be noted that the network meta-analysis established that the OR of mortality with full-dose thrombolysis compared with anticoagulation was 0.60 (95\% CI 0.36 to 1.01 ). It is possible that better patient selection with a higher risk of adverse outcomes and a lower risk of bleeding may allow full-dose thrombolysis to confer a benefit if trials were to be performed in these groups (to be defined). Low-dose and catheter-directed thrombolysis compared with anticoagulation had OR of 0.47 (0.14 to 1.59 ) and 0.31 (0.01 to 7.96), respectively. Major bleeding appeared to be lowest in the low-dose thrombolysis group, followed by catheter-directed thrombolysis and finally fulldose thrombolysis.

These findings are not definitive but are helpful. It is unlikely that the next iteration of PE guidelines will be significantly influenced by this paper to the extent of making a new recommendation, since the level of evidence is not strong enough. We will probably still be left with an option to 'consider' reperfusion therapy in some form of increased-risk subgroup of normotensive patients. Our unit receives a number of calls for advice on what to do with normotensive patients with PE who display many adverse risk factors, such as elevated biomarkers and large thrombus burden, including residual proximal deep vein thrombosis and free-floating rightsided cardiac chamber thrombus; thus, many clinicians appreciate the need for therapy that is more aggressive than anticoagulation in a small, but difficult to define, group of non-high-risk patients. We cannot always expect to find the answer in clinical guidelines to every problem that front-line medicine throws at us. The findings from the study of Jimenez et al may well assist us when we 'consider' what to do with patients in this 'grey zone' of increased risk.

It is not surprising that we are still left with many questions in relation to reperfusion 
therapy in PE, although with studies such as that of Jimenez et al it does feel as if we are edging closer to knowing how to use it. There is still a need to define the group of patients who will benefit from reperfusion therapy, and if the previous studies were not already enough of a springboard to design and run a large trial with reduced-dose thrombolysis, then the study by Jimenez et al ought to be. The only stumbling block will be if further risk stratification of patients results in difficulty recruiting to a large enough study, although in theory a higher risk group would reduce the sample size needed to see benefit. If the outcomes were to show significant improvement in outcomes with a low bleeding risk, then this would become the new gold standard against which to test catheter-directed thrombolysis, which at present is still trying to find its rightful home.

One of the justifications for thrombolysis in the past was to prevent the serious long-term outcome of chronic thromboembolic pulmonary hypertension. At least for now, the extension study of PEITHO has settled that argument with no clear long-term benefit from thrombolysis. ${ }^{9}$ So we are getting there, but slowly...
Competing interests LSH received honoraria for scientific advice as part of a trial steering committee from BTG plc.

Provenance and peer review Commissioned; externally peer reviewed.

(C) Article author(s) (or their employer(s) unless otherwise stated in the text of the article) 2018. All rights reserved. No commercial use is permitted unless otherwise expressly granted.

\section{A) Check for updates}

To cite Howard LS. Thorax 2018;73:412-413.

Published Online First 4 January 2018

\section{SLinked}

- http://dx.doi.org/10.1136/thoraxjnl-2017-210040

Thorax 2018;73:412-413.

doi:10.1136/thoraxjnl-2017-211141

\section{REFERENCES}

1 NICE. Venous thromboembolic diseases: the management of venous thromboembolic diseases and the role of thrombophilia testing. London, UK: National Clinical Guideline Centre, 2012.

2 Konstantinides SV, Torbicki A, Agnelli G, et al. 2014 ESC guidelines on the diagnosis and management of acute pulmonary embolism. Eur Heart J 2014;35:3033-69.
3 Meyer G, Vicaut E, Danays T, et al. Fibrinolysis for patients with intermediate-risk pulmonary embolism. N Engl J Med 2014;370:1402-11.

4 Becattini C, Agnelli G, Lankeit M, et al. Acute pulmonary embolism: mortality prediction by the 2014 European Society of Cardiology risk stratification model. Eur Respir J 2016;48:780-6.

5 Jiménez D, Kopecna D, Tapson V, et al. Derivation and validation of multimarker prognostication for normotensive patients with acute symptomatic pulmonary embolism. Am J Respir Crit Care Med 2014;189:718-26.

6 Jiménez D, Aujesky D, Moores L, et al. Combinations of prognostic tools for identification of high-risk normotensive patients with acute symptomatic pulmonary embolism. Thorax 2011;66:75-81.

7 Chatterjee S, Chakraborty A, Weinberg I, et al. Thrombolysis for pulmonary embolism and risk of all-cause mortality, major bleeding, and intracranial hemorrhage: a meta-analysis. JAMA 2014;311:2414-21.

8 Jimenez D, Martin-Saborido C, Muriel A, et al. Efficacy and safety outcomes of recanalisation procedures in patients with acute symptomatic pulmonary embolism: systematic review and network meta-analysis. Thorax 2018;73:464-71.

9 Konstantinides SV, Vicaut E, Danays T, et al. Impact of Thrombolytic Therapy on the Long-Term Outcome of Intermediate-Risk Pulmonary Embolism. J Am Coll Cardiol 2017;69:1536-44 